\title{
MICROWAVE RADAR IMAGING OF INHOMOGENEOUS BREAST PHANTOMS USING CIRCULAR HOLOGRAPHY
}

\author{
Daniel Flores-Tapia ${ }^{1,2}$, Oleksandr Maizlish ${ }^{2}$, Clive Alabaster ${ }^{3}$ and Stephen Pistorius $^{1,4,5}$ \\ ${ }^{1}$ Division of Medical Physics, CancerCare Manitoba, Winnipeg, MB, Canada \\ ${ }^{2}$ Department of Mathematics, University of Manitoba, Winnipeg, MB, Canada \\ ${ }^{3}$ Department of Informatics and Systems Engineering, Cranfield University, Shrivenham, UK \\ ${ }^{4}$ Department of Physics and Astronomy, University of Manitoba,Winnipeg MB, Canada \\ ${ }^{5}$ Department of Radiology, University of Manitoba, Winnipeg, MB, Canada
}

\begin{abstract}
Circular holography is a novel reconstruction technique for Breast Microwave Radar (BMR) imaging. Compared to current state of the art BMR image formation methods, this reconstruction approach yields spatially accurate images with higher signal to noise ratios and no artifacts. Nevertheless, a preclinical study is required to assess the feasibility of this technique in realistic breast imaging scenarios. In this paper, a series of preliminary results showing the performance of circular holography on preclinical datasets are presented. These datasets were recorded from inhomogeneous breast phantoms that mimic the dielectric properties and the anatomy of the different breast tissues. These phantoms were fabricated using Magnetic Resonance (MR) images a base model to emulate the shape and volumes of dense tissue regions. The reconstructed BMR images show that tumor and fibroglandular tissue responses can be effectively distinguished, suggesting that circular holography can be used as BMR reconstruction approach in clinical scenarios.
\end{abstract}

Index Terms - Breast microwave radar, image reconstruction, preclinical feasibility.

\section{INTRODUCTION}

In 2008, breast cancer comprised $22.9 \%$ of all cancer related diagnosis around the world and over 200,000 persons died from it [1]. As with many other types of cancer, early stage detection and treatment are crucial to increase the patient's chances for survival. Currently, x-ray mammography is the gold standard for breast cancer screening. Nevertheless, this technology has a sensitivity of $92 \%$ and a specificity between $40-70 \%$, allowing room for improvement. During the last decade, the use of Breast Microwave Radar (BMR) techniques has attracted the attention of the medical imaging community as a complimentary technique for breast cancer detection [2,3]. This technology is based on the significant contrast between the dielectric properties of healthy breast tissues and malignant lesions, which is at least 1.5-2:1 according to the latest in vivo studies $[4,5]$. Recent studies have shown that BMR can generate high contrast images and detect tumors of at least $10 \mathrm{~mm}$ [3]. Although BMR techniques have been tested in small scale clinical trials, this technology must be optimized so it can be used on routine basis [4].

A factor that can noticeably affect the performance of BMR is the amount of dense tissue in the breast region [3]. Depending on the location and size of the fibroglandular tissue regions, the responses from these structures can noticeably reduce the contrast in BMR images. Since the $95 \%$ of tumors grow in very close proximity of dense tissue structures [6], the performance of a BMR reconstruction method must be tested under this consideration to evaluate its clinical feasibility.

Circular holography is a novel BMR reconstruction approach that process the spectrum of the recorded breast responses and eliminates the phase wrappings introduced by the scan geometry. This procedure yields spatial accurate images that show the locations and dimensions of the inclusions present inside the scan region [7]. Compared to time-domain BMR reconstruction approaches, images formed using circular holography have a higher Signal to Noise Ratio (SNR), focal quality and do not contain any high magnitude artifacts.

In this paper, the experimental feasibility of circular holography on scan scenarios with fibroglandular tissue structures was assessed. Compared to previous studies using similar phantoms [8], the fibroglandular structures used in this work have irregular morphologies that mimic the shape of realistic dense tissue regions. These datasets were recorded from phantoms that closely mimic the dielectric properties of breast tissues using a preclinical BMR system. The anatomy of the phantoms was based on Magnetic Resonance (MR) images in order to recreate the morphological patterns that may cause high scattering, such as sharp corners. 


\section{METHODOLOGY}

The phantom materials used in this study mimic the actual dielectric properties of the breast tissues, instead of only emulating their dielectric contrast. Since the propagation losses and skin reflections strongly affect the amplitude of the recorded responses, they need to be accurately recreated. The phantom was created using a styrene-acrylonitril cylinder with a diameter of $13 \mathrm{~cm}$ and a height of $35 \mathrm{~cm}$. Fatty breast tissue was simulated using glycerin, which closely emulates the dielectric properties of mimics the dielectric properties of fatty breast tissues [4]. Tumors were simulated using capsules filled with an $85 \%$ distilled water and $15 \%$ fructose solution. The dielectric characteristics of this mixture are described in detail in a study performed by Shinyashiki et al. [9]. All the tumors used in the experiments presented in this paper had a hemispherical shape. The authors also considered spiculated tumor morphologies, and although this morphology presented responses with a higher magnitude in some cases, the reconstructed images presented less consistency than its spherical counterparts. Fibroglandular tissue structures and skin were emulated using a mixture of a synthetic polymer (TX-151), polyethylene powder and distilled water. The dielectric properties of this mixture can be adjusted by varying the amount of water. The final composition of the TX151, polyethylene and water mixture used in this study had similar properties to the dielectric properties of fibroglandular tissues reported in [4,5]. A $2 \mathrm{~mm}$ layer of synthetic skin was attached to the cylindrical container. The dielectric properties of the matching liquid and the phantom materials are given in Table I. A picture of the data acquisition setup is shown in figure 1 .

The BMR datasets used in this study were recorded using a preclinical data acquisition system. This system is formed by a $56 \times 57.4 \times 40 \mathrm{~cm}$ acrylic enclosure a lid that serves a support structure for a Vivaldi antenna and a rotating platform that holds the synthetic dense tissue and tumor structures. The tank was filled with canola oil to provide impedance matching between the antenna and the breast phantom [2]. The dielectric characteristics of the canola oil are described in a study performed by Fear et al. [2]. All the experiments were recorded using a monostatic scan protocol, in which the same antenna is used to transmit and receive. This scan methodology has proven to be more robust compared to multiscatic protocols, where different pairs of receiver and transmitter antennas are used [10].

The responses from the phantom were recorded using an Anritsu MS2026A vector network analyzer, which functioned both as the waveform generator and recording device. The Vivaldi antenna, manufactured using 2 layers of Arlon-Diclad 527 with permittivity 2.65 and loss tangent of 0.0022 , was connected to the VNA via a $50 \Omega$ cable and mounted into a waterproof acrylic mounting structure. The antenna has a bandwidth of $5 \mathrm{GHz}$ with a center frequency of $3.5 \mathrm{GHz}$. Its dimensions are $12 \times 10.5 \mathrm{~cm}$. The data acquisition setup has an acrylic lid which has a series of support structures for the antenna holder and platform in which a stepper motor with a precision of .005 degrees per step. This motor is attached to a support structure in which acrylic rods are inserted. These rods are used to hold the synthetic fibroglandular and tumor structures into their positions inside the phantom. The authors evaluated the effect of these rods on the reconstructed datasets by evaluating 32 datasets collected with 3 to 5 rods inside and comparing them to datasets from phantoms that only contained glycerine. No significant differences were found.

TABLE I.

Dielectric properties of the materials used in the phantom.

\begin{tabular}{|l|c|c|}
\hline \multicolumn{1}{|c|}{ Material } & $\boldsymbol{\varepsilon}_{\mathbf{r}}(\mathbf{3 G H z})$ & $\sigma(\mathbf{3 G H z})$ \\
\hline Skin & 35 & $1.2 \mathrm{~S} / \mathrm{m}$ \\
\hline $\begin{array}{l}\text { Skin polymer- } \\
\text { distilled water } \\
\text { mixture }\end{array}$ & 32 & $1.1 \mathrm{~S} / \mathrm{m}$ \\
\hline $\begin{array}{l}\text { Low density } \\
\text { Breast tissue }\end{array}$ & 9.9 & $.72 \mathrm{~S} / \mathrm{m}$ \\
\hline Glycerin & $25-37$ & $1.5 \mathrm{~S} / \mathrm{m}$ \\
\hline $\begin{array}{l}\text { Fibroglandular } \\
\text { tissue }\end{array}$ & 35 & $1.6 \mathrm{~S} / \mathrm{m}$ \\
\hline $\begin{array}{l}\text { Dense polymer- } \\
\text { distilled water } \\
\text { mixture }\end{array}$ & & $2.1 \mathrm{~S} / \mathrm{m}$ \\
\hline Tumor & 55 & $1.9 \mathrm{~S} / \mathrm{m}$ \\
\hline $\begin{array}{l}\text { Fructose-saline } \\
\text { mixture }\end{array}$ & 2.5 & $.035 \mathrm{~S} / \mathrm{m}$ \\
\hline Canola oil & 2.6 & $.001 \mathrm{~S} / \mathrm{m}$ \\
\hline Styrene-acrylonitrile
\end{tabular}

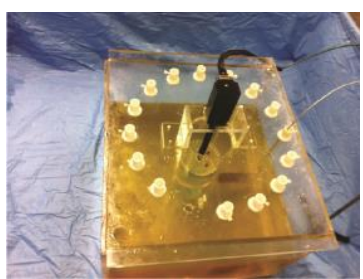

a)

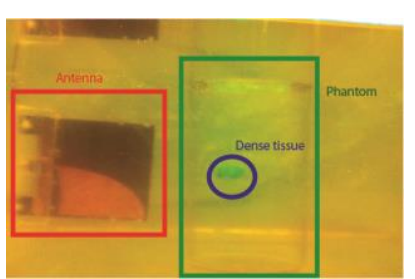

b)
Figure 1. a) Top view of the preclinical data acquisition setup, b) Lateral view of the antenna and a phantom containing a synthetic fibroglandular tissue structure.

The platform was rotated to emulate a circular scan geometry with a radius of $8.25 \mathrm{~cm}$. The phantom was positioned in the center of the tank and the platform holding the fibroglandular tissue and tumor structures was rotated to perform the scan process performed described in [7]. In the experiments, the platform holding the structures inside the phantom was rotated in $5^{\circ}$ increments, which satisfies the 
sampling criterion proposed in [7] which is required to avoid aliasing. At each scan location a stepped frequency continuous wave with a bandwidth of $5 \mathrm{GHz}$ and a center frequency of $3.5 \mathrm{GHz}$ was radiated. Each waveform had 551 frequency points. The power of each frequency component in the irradiated wave was $0 \mathrm{dBm}$. To eliminate the tank reflections from the recorded datasets, a calibration signal was obtained by averaging a set of 64 responses of the system with no phantom present. This signal was subtracted from the recorded datasets to extract the phantom responses.

\section{RESULTS}

The results presented in this paper were obtained from two experimental datasets were recorded from phantoms with a scattered fibroglandular breast density (ACR2). The morphology of the phantoms used in this study was modeled based on a series of breast MR images obtained from the University of Wisconsin breast image repository. In both experimental setups, a hemispherical tumor with a diameter of $6 \mathrm{~mm}$ was placed next to a fibroglandular tissue structure to recreate the location malignant lesions in a realistic clinical scenario. This was done since over $90 \%$ of all breast tumors grow in the immediate vicinity of dense tissue structures, such as milk ducts or lobules. All the datasets were reconstructed using the holographic BMR proposed by the authors in [7]. All the synthetic fibroglandular tissue structures used did not extend through the whole length of the phantom, and their volume and shape was obtained from the MR base image. The datasets were processed using a PC with a Pentium i7 processor and 12GB of RAM. This workstation contains three NVidia Tesla General Purpose Graphic Processing Units (GPGPUs) that were used to reconstruct the recorded datasets. Using this computing platform, the reconstruction process took in average 5.01 milliseconds.

The performance of the proposed approach was evaluated by calculating the SNR, contrast to noise ratio (CNR) of the reconstructed images and the spatial error of the reconstructed tumor locations. The SNR and the CNR were calculated as follows:

$$
\begin{gathered}
S N R=20 \cdot \log _{10}\left(-\frac{}{b}\right) \\
C N R=10 \cdot \log _{10}\left(\frac{2 r^{2}}{2}\right)
\end{gathered}
$$

where is the magnitude of the tumor signature, is the magnitude of the largest magnitude of the fibroglandular structures signature and $\quad b$ is the standard deviation of the background. This definition was adopted because the energy of the reconstructed images is commonly used to assess the presence of any lesions [2,3].

The MR image used to create the first phantom and its corresponding BMR image are shown in figure 2 a) and b) respectively. In this setup the tumor was positioned at the fibroglandular structure at the upper region of the breast. To improve the visualization of the BMR image, the skin response was removed using the approach proposed by the authors in [7] and its location is indicated by the white circle. Notice how the location of the tumor in the reconstructed BMR image is consistent with its location on the experimental setup. The power of the reconstructed tumor signature is also more than twice than the magnitude of the synthetic fibroglandular tissue structures.

The MR image used as a model for the second phantom is shown in figure 3 a). In this setup, the tumor was positioned next to a dense tissue structure at $(1.9,-2.8) \mathrm{cm}$ in the lower section of the MR image. The reconstructed BMR image is shown in figure $3 \mathrm{~b}$ ). Similarly to the previous experiment, the location of the reconstructed tumor response is consistent with its location in the experimental setup. The reconstructed tumor response also has the highest power values in the image. A summary of the SNR, CNR and spatial error values obtained in each experiment is shown in Table II.

TABLE II.

SNR, CNR and spatial error values of each experiment.

\begin{tabular}{|l|l|l|l|}
\hline Experiment/Metric & SNR & CNR & $\begin{array}{l}\text { Spatial } \\
\text { accuracy }\end{array}$ \\
\hline 1 & $7.69 \mathrm{~dB}$ & $4.70 \mathrm{~dB}$ & $(1,0) \mathrm{mm}$ \\
\hline 2 & $7.51 \mathrm{~dB}$ & $3.73 \mathrm{~dB}$ & $(3,0) \mathrm{mm}$ \\
\hline
\end{tabular}

\section{CONCLUSION}

In this paper, the preclinical feasibility of circular holographic BMR reconstruction approaches was assessed using inhomogeneous breast phantoms. These phantoms were created using materials that closely mimic the dielectric properties of fatty, fibroglandular and malignant breast tissues. MR images were used as a model for the location and morphologies of the dense tissue structures. The performance of circular holography was measured by calculating the SNR, CNR of the reconstructed images and the spatial accuracy of the tumor location.

The reconstructed tumor responses were consistent with its locations in the experimental setups. Compared to previous BMR studies performed on inhomogeneous phantoms [3], the images generated using circular holography exhibited similar spatial accuracy and higher SNR values. The reconstructed BMR images presented $\mathrm{SNR}$ and $\mathrm{CNR}$ values above $7.5 \mathrm{~dB}$ and $3.7 \mathrm{~dB}$ respectively, which indicates that tumors responses can be distinguished from fibroglandular tissue reflections, even in scenarios where tumors are located next to dense tissue structure. Future research will be 
focused on further validating these findings using breast phantoms with a hemispherical shape.

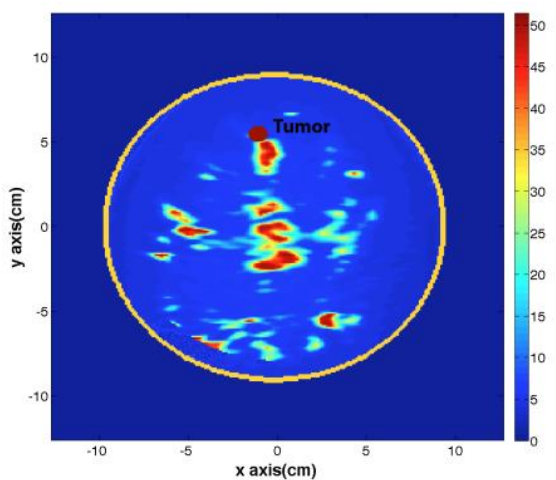

a)

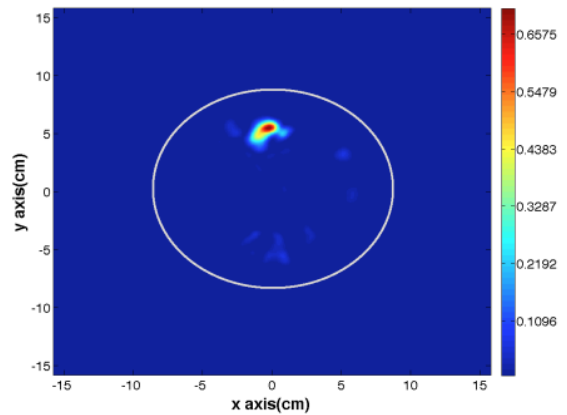

b)

Figure 2. a) MR image used to as a basis for the first experimental setup, b) Reconstructed BMR image

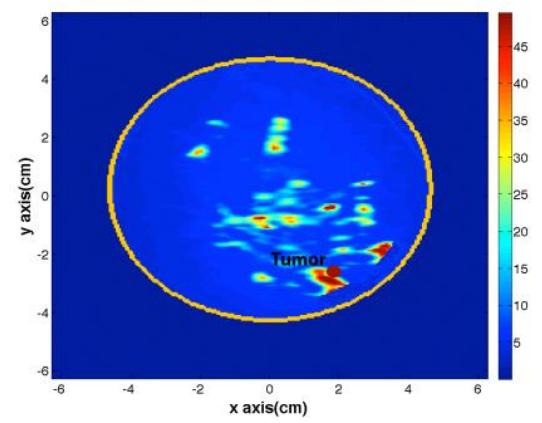

a)

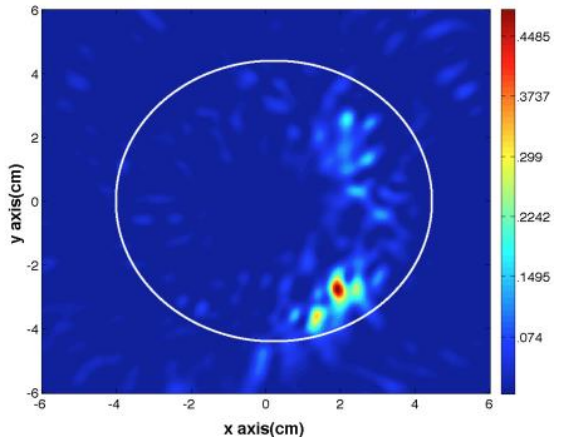

b)

Figure 3. a) MR image used to as a basis for the second experimental setup, b) Reconstructed BMR image

\section{REFERENCES}

[1] International Agency for Research on Cancer, World Breast Cancer Report 2008, World Health Organization, Lyon, France, 2008.

[2] J.M. Sill and E.C. Fear, "Tissue sensing adaptive radar for breast cancer detection - experimental investigation of simple tumor models," IEEE Trans. Microwave Theory and Techniques, vol. 53, pp. 3312-3319, November 2005.

[3] M. Klemm, J.A. Leendertz, D. Gibbins, I.J. Craddock, A. Preece and R. Benjamin, "Microwave Radar-Based Breast Cancer Detection: Imaging in Inhomogeneous Breast Phantoms", IEEE Antennas and Wireless Propagation Letters, vol.8, pp-1349-1352, January 2010.

[4] P.M. Meaney, M.W. Fanning MW, T. Raynolds, C.J. Fox, Q. Fang, C.A. Kogel, S.P. Poplack and K.D. Paulsen, "Initial clinical experience with microwave breast imaging in women with normal mammography," Academic Radiology, vol. 14, pp. 207-218, March 2007.

[5] R.J. Halter, T. Zhou, P.M. Meaney, A. Hartov, R. Barth, K. Rosenkranz, W. Wells, C. Kogel, A. Borsic, E. Rizzo and K.D. Paulsen, "The correlation of in vivo and ex vivo tissue dielectric properties to validate electromagnetic breast imaging: initial clinical experience," Physiological Measurement, vol. 30, pp. S121-S136, June 2009.

[6] L.L. Hughes , M. Wang , D.L. Page , R. Gray , L.J. Solin, N.E. Davidson , M.A. Lowen, J.N. Ingle, A. Recht, W.C. Wood," Local excision alone without irradiation for ductal carcinoma in situ of the breast: a trial of the Eastern Cooperative Oncology Group," Journal of Clinical Oncology, vol. 27, pp. 5319-24, November 2009.

[7] D. Flores-Tapia, G. Thomas and S. Pistorius, "A Wavefront Reconstruction Method for 3D Cylindrical Subsurface Radar Imaging," IEEE Transactions on Image Processing, vol. 17, pp. 1908-1925, October 2008.

[8] D. Flores-Tapia, G. Thomas and S. Pistorius, "Real time breast microwave radar image reconstruction using circular holography: A study of experimental feasibility," Medical Physics, in press, published online September 2011.

[9] N. Shinyashiki, M. Shinohara, Y. Iwata, T. Goto, M. Oyama, S. Suzuki, W. Yamamoto, S. Yagihara, T. Inoue, S. Oyaizu, S. Yamamoto, K. L. Ngai and S. Capaccioli, "The Glass Transition and Dielectric Secondary Relaxation of FructoseWater Mixtures," Journal of Physical Chemistry B, vol. 112, 15470-15477, December 2008.

[10] D. Byrne, M. O'Halloran, M. Glavin, and E. Jones, " Data Independent Radar Beamforming Algorithms for Breast Cancer Detection", Progress In Electromagnetics Research, vol. 107, pp. 331-348, 2010. 